\title{
STATIONARY WAVES FORCED BY TOPOGRAPHY IN A VERTICALLY SHEARED, STRATIFIED, ROTATING FLUID
}

\author{
C, B. FANDRY, R. L. HUGHES and L. M. LESLIE'
}

(Received 8 April 1982; revised 6 August 1982)

\begin{abstract}
The effect of an isolated topographic bump in a two-layer fluid on a $\beta$-plane is investigated. An analytical solution is derived in terms of the appropriate Green's function for arbitrary topography of finite horizontal extent. It is found that the disturbances generated by the bump are composed of two fundamental modes which may be wave-like or evanescent. The wave-like modes are topographically induced Rossby waves which occur only when there is eastward flow in at least one of the layers. These waves are always confined to the downstream (eastward) side of the bump. Whereas previous studies of this type have concentrated on eastward flow over topography, the theory has been extended here to include a wide range of vertically sheared flows. Particularly important is the case of low level westward flow combined with upper level eastward flow, as it has direct application, for example, to the summertime atmospheric circulation over the sub-tropical regions of the continental land.masses. In this case a wave-like disturbance extends far downstream from the bump for sufficiently large shear, and is of smaller amplitude in the upper layer than in the lower layer because of the effects of the stratification. For small shears, the wave-like mode in the lower layer is small and the character of the disturbance is evanescent, confining it to the immediate neighbourhood of the bump. A stability analysis of the solutions shows that the disturbances may be baroclinically unstable for sufficiently large mean shear.
\end{abstract}

\section{Introduction}

Since the early work on Taylor columns [15], the geophysical problem of ocean currents flowing over bottom topography or atmospheric winds blowing over orography has been the subject of numerous theoretical and experimental studies. Most of these studies have concentrated on motions with large time scales compared with the time scale associated with the earth's rotation $\left(f_{0}^{-1}\right)$, space

\footnotetext{
' Australian Numerical Meteorology Research Centre, P.O. Box 5089AA, Melbourne, Victoria 3001. (c) Copyright Australian Mathematical Society 1983
} 
scales, $L$, much larger than the depth of the fluid, $H$, and velocity scale, $\bar{U}$, such that the Rossby number, $\varepsilon=\bar{U} / f_{0} L$, is very small. In addition, the topographic height scale, $h$, is much smaller than $H$, and the ratio $h / H$ is similar in magnitude to the Rossby number. Some studies have also taken into account the effects of vertical density stratification. These have been incorporated by considering a density structure which varies either continuously with depth or discretely in a finite number of layers of known density and depth. On the hasis of these scales of motion the well known quasi-geostrophic potential vorticity equation was formally derived by Pedlosky [11] for both a continuously stratified fluid and a layered fluid. Many different oceanic and atmospheric phenomena, particularly those associated with Rossby and topographic waves, have been explained within the framework of quasi-geostrophic motion.

The particular problem of interest here is the influence of a finite ridge or bump on a vertically stratified, rotating, sheared flow. This problem has been treated by a number of authors, and some of the contributions have been summarized by McCartney [8]. Using a two-layer model, McCartney [8] showed that the $\beta$-effect limited the horizontal influence of the topography for westward (retrograde) flow. For eastward (prograde) flow over a topographic ridge McCartney [8], [9] showed that a pattern of stationary Rossby waves would form downstream (to the east) of the ridge. In the case of an infinite ridge these waves would extend to downstream infinity, whereas for a finite ridge they would decrease as $O\left(r^{-1 / 2}\right)$, where $r$ is the horizontal distance from the ridge.

In the context of the two-layer model, the influence of a counter-flow situation, with eastward flow in one layer and westward in the other, has received very little attention. However, counter-flow situations of great interest do occur naturally in the atmosphere; for example, flow in the subtropics over the Australian (and other continental) land masses during the summer period where low level westward flow is surmounted by upper level eastward flow. The interaction between this counter-flow wind regime and the main topographic features, namely the eastern highlands and the Western Australia plateau, is largely responsible for the variety of low-level pressure trough formations that are observed [4].

In this paper a two-layer model is used to describe the finite-amplitude, steady disturbances caused by finite topographic features to a horizontally uniform, vertically sheared flow. Previous solutions have been restricted to those applying to very simple topographic forms such as step functions ([14], [8], [9]) and other symmetric forms (e.g. [2], [3]). In this work, we present a generalized solution of the quasi-geostrophic potential vorticity equation in terms of the appropriate Green's function. Solutions for arbitrarily shaped topography are then determined and illustrated for different basic flow configurations. The previously neglected situation of upper and lower layer basic flows in opposite directions is carefully examined, and some interesting features are found. 
In addition a stability analysis of the solutions reveals that for sufficiently large vertical shear the basic flows may be baroclinically unstable to disturbances of large enough scale. The various parameter ranges under which this occurs are presented.

\section{Basic equations and boundary conditions}

The model, illustrated in Figure 1 consists of two well mixed layers of constant density (potential temperature in the atmosphere) $\rho_{1}, \rho_{2}$ where the subscripts 1 and 2 refer to the upper and lower layers respectively. The upper surface is rigid and the undisturbed depths of the upper and lower layers are $D_{1}$ and $D_{2}-$ $h^{\prime}(x, y)>0$ respectively, where $x$ and $y$ are horizontal cartesian co-ordinates. For topographic bumps of finite horizontal extent, $h^{\prime} \rightarrow 0$ as $x^{2}+y^{2} \rightarrow \infty$, the flow in each layer is taken to be uniform and zonal and equal to $U_{1}^{\prime}, U_{2}^{\prime}$ far upstream and downstream of the bump.

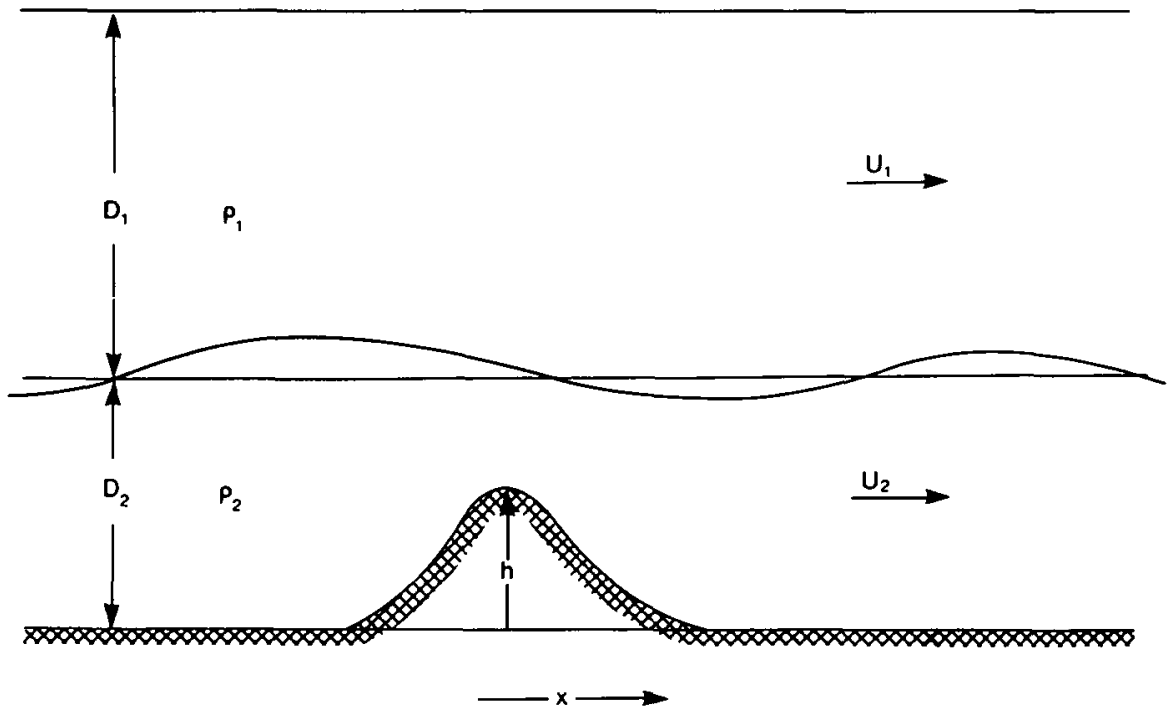

Figure 1. Zonal section of two-layer model: $x$ is zonal, $y$ is meridional.

The well known form of the quasi-geostrophic potential vorticity equations for such a two-layer system may be written as

$$
\begin{gathered}
\mathscr{D}_{1} / \mathscr{D}_{t}\left[\nabla^{2} \psi_{1}-F_{1}\left(\psi_{1}-\psi_{2}\right)+\beta y\right]=0, \\
\mathscr{D}_{2} / \mathscr{D}_{t}\left[\nabla^{2} \psi_{2}-F_{2}\left(\psi_{2}-\psi_{1}\right)+\beta y+h\right]=0 .
\end{gathered}
$$


These are the non-dimensional equations in which $\mathscr{D}_{t} / \mathscr{D}_{t}$ is the substantial

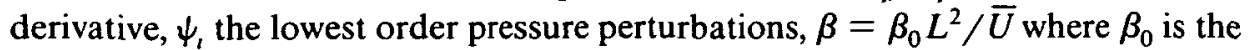
(dimensional) northward gradient of the Coriolis parameter and $\nabla^{2}=\partial^{2} / \partial x^{2}+$ $\partial y^{2}$. The non-dimensional topography $h(x, y)$ is given by

$$
h=h^{\prime} / \varepsilon D_{2},
$$

where $\varepsilon$ is the Rosshy number previously defined. The constants $F_{1}$ and $F_{2}$ are defined by

$$
F_{1}=\frac{f_{0}^{2} L^{2}}{g^{\prime} D_{1}}, \quad F_{2}=\frac{f_{0}^{2} L^{2}}{g^{\prime} D_{2}},
$$

where $g^{\prime}$ is the reduced gravity defined by $g^{\prime}=2 g\left(\rho_{2}-\rho_{1}\right) /\left(\rho_{1}+\rho_{2}\right)$. In physical terms these constants represent the square of the ratio of the geometric Rossby height to the depths of the layers. Details of the derivation of equations (2.1 a, b) and of the associated non-dimensionalization may be found, for example, in [12]. The most important assumptions are that the Rossby number, $\varepsilon$, and the aspect ratio $\left(D_{1}+D_{2}\right) / L$ are small.

Perturbations of the upper layer flow are caused by vortex compression and stretching produced by distortions of the interface. The dimensional amplitude of the interface perturbation is given by

$$
\zeta=\varepsilon D_{2} F_{2}\left(\psi_{2}-\psi_{1}\right),
$$

and $\psi_{1}, \psi_{2}$ may be considered as streamfunctions for the upper and lower layers respectively.

The topography is assumed to be horizontally finite, i.e.,

$$
h \rightarrow 0 \text { as } x^{2}+y^{2} \rightarrow \infty,
$$

and uniform far upstream and downstream flows in each layer imply

$$
\psi_{1} \rightarrow-U_{1} y \text { and } \psi_{2} \rightarrow-U_{2} y \text { as } x^{2}+y^{2} \rightarrow \infty,
$$

where

$$
U_{1}=U_{1}^{\prime} / \bar{U} \text { and } U_{2}=U_{2}^{\prime} / \bar{U}
$$

Assuming steady flow, equations (2.1) may be integrated along streamlines with constants of integration determined from the far field. For sufficiently large values of $h$ it may be anticipated ([5], [6], [7]) that closed streamlines will occur in the vicinity of the topographic feature, in which case the far field flow can no longer be used to determine the constants along these streamlines. This situation will be avoided in this study.

In terms of the perturbation streamfunctions,

$$
\phi_{i}=\psi_{i}+U_{i} y \quad \text { for } i=1,2,
$$


equations (2.1) then become

$$
\begin{gathered}
\nabla^{2} \phi_{1}+F_{1} \phi_{2}+\beta y=\gamma_{1} \phi_{1}-\left(F_{1} U_{2}-\gamma_{1} U_{1}\right) y, \\
\nabla^{2} \phi_{2}+F_{2} \phi_{1}+\beta y+h=\gamma_{2} \phi_{2}-\left(F_{2} U_{1}-\gamma_{2} U_{2}\right) y,
\end{gathered}
$$

where

$$
\gamma_{1}=\left(F_{1} U_{2}-\beta\right) / U_{1} \text { and } \gamma_{2}=\left(F_{2} U_{1}-\beta\right) / U_{2},
$$

and $\phi_{1}$ satisfy the conditions

$$
\phi_{t} \text { bounded and } \phi_{t} \rightarrow 0 \text { as } x^{2}+y^{2} \rightarrow \infty \text {. }
$$

These equations are equivalent to equations (2.16) of McCartney [9], who eliminated $\phi_{1}$ to form a fourth order partial differential equation for $\phi_{2}$. Realizing that the flow in each layer must be comprised of a barotropic and a baroclinic mode, it is mathematically simpler to combine equations $(2.8 \mathrm{a}, \mathrm{b})$ into the second order normal mode equation

$$
\nabla^{2} \Phi-\lambda \Phi=-b(x, y)
$$

where $b=\alpha h$, and

$$
\lambda=-\alpha F_{2}+\gamma_{1}
$$

The normal modes are given by

$$
\Phi^{(\imath)}=\phi_{1}+\alpha^{(\imath)} \phi_{2}, \quad i=1,2,
$$

where $\alpha^{(t)}, i=1,2$ satisfy the quadratic equation

$$
\alpha^{2}-\alpha\left(\gamma_{1}-\gamma_{2}\right) / F_{2}-F_{1} / F_{2}=0,
$$

with roots

$$
\alpha^{(1),(2)}=\left(\gamma_{1}-\gamma_{2}\right) / 2 F_{2} \pm\left[\left(\left(\gamma_{1}-\gamma_{2}\right) / 2 F_{2}\right)^{2}+F_{1} / F_{2}\right]^{1 / 2} \text {. }
$$

Expressions for the perturbation streamfunctions in terms of the two normal modes are easily found from (2.13) to be

$$
\begin{gathered}
\phi_{1}=\left(\alpha^{(1)} \Phi^{(2)}-\alpha^{(2)} \Phi^{(1)}\right) /\left(\alpha^{(1)}-\alpha^{(2)}\right), \\
\phi_{2}=\left(\Phi^{(1)}-\Phi^{(2)}\right) /\left(\alpha^{(1)}-\alpha^{(2)}\right) .
\end{gathered}
$$

We shall later identify $\phi^{(2)}$ with the barotrophic mode and $\phi^{(1)}$ with the baroclinic mode.

Solutions of the non-homogeneous Helmholtz equation (2.11) are evanescent if $\lambda>0$ and wave-like if $\lambda<0$. The wave-like solutions of equation (2.11) have been discussed in other geophysical contexts such as lee waves in a stratified fluid [10] and stationary Rossby waves [9].

If $\lambda>0$, the conditions (2.10) are sufficient to determine uniquely the evanescent solution of equation (2.11). But if $\lambda<0$, the wave-like solutions are not 
uniquely determined by condition (2.10) alone, and a radiation condition preventing "upstream" waves must be added. This is of the form

$$
\left(x^{2}+y^{2}\right)^{1 / 4} \Phi \rightarrow 0, \quad \text { far "upstream". }
$$

The relevant quantity determining "far upstream" is the east-west group velocity for each stationary mode of wave number $k, d \omega^{(t)} /\left.d k\right|_{\omega^{(1)}=0}$, where

$$
\begin{aligned}
& \omega^{(i)} / k=U_{2}+\left[U_{s} k^{2}\left(k^{2}+2 F_{2}\right)-\beta\left(2 k^{2}+F_{1}+F_{2}\right) / 2 k^{2}\left(k^{2}+\underline{F}_{\mathrm{i}}+\underline{F}_{2}\right)\right] \\
& \pm\left[\beta^{2}\left(F_{1}+F_{2}\right)^{2}+2 \beta U_{2} k^{4}\left(F_{1}-F_{2}\right)\right. \\
& \left.-k^{4} U_{s}^{2}\left(4 F_{1} F_{2}-k^{4}\right)\right]^{1 / 2} / 2 k^{2}\left(k^{2}+F_{1}+F_{2}\right), \quad i=1,2,
\end{aligned}
$$

where $U_{s}=U_{1}-U_{2}$. This expression is easily found by looking for the plane wave solutions of the time dependent linearized equations equivalent to (2.1). McCartney [8] states that the group velocity may be eastward or westward, in which case "upstream" will mean $x \rightarrow-\infty$ or $x \rightarrow+\infty$ respectively. When the flow in both layers is eastward $\left(U_{1}>0, U_{2}>0\right)$, it can be shown analytically that the group velocity for each mode is always eastward and condition (2.17) is applied as $x \rightarrow-\infty$.

The group velocity for each mode in the counter-flow situation is not easily determined and must be evaluated numerically in each individual case. Without loss of generality, the specific cases considered here were chosen so that the group velocities of the stationary waves are always eastward, and hence condition (2.17) will always apply as $x \rightarrow-\infty$.

The two-dimensional boundary value problem in the region $-\infty<x<\infty$, $-\infty<y<\infty$ represented by equations (2.10), (2.11) and (2.17) for an arbitrarily shaped topography $h(x, y)$ may be formulated in terms of the appropriate Green's function satisfying

$$
\nabla^{2} G-\lambda G=-\delta(x-\xi, y-\eta),
$$

with $G$ bounded except at the point $(\xi, \eta)$

and $G \rightarrow 0$ as $x^{2}+y^{2} \rightarrow \infty$.

$$
\text { For } \lambda<0, \quad r^{1 / 2} G \rightarrow 0 \text { as } x \rightarrow-\infty,
$$

where $r^{2}=(x-\xi)^{2}+(y-\eta)^{2}$ and $\delta$ is the Dirac delta function. Since $G$ satisfies the same equation and boundary conditions as $\Phi$, the solution may be written as

$$
\Phi(x, y)=\alpha \int_{-\infty}^{\infty} d \xi \int_{-\infty}^{\infty} h(\xi, \eta) G(x, y ; \xi, \eta) d \eta .
$$

For $\lambda>0$, it is easily shown that the Green's function satisfying equations (2.18) is radially symmetric and given by

$$
G(x, y ; \xi, \eta)=\frac{1}{2 \pi} K_{0}\left(\lambda^{1 / 2} r\right)
$$


where $K_{0}$ is the zeroth order modified Bessel function of the second kind whose properties may be found in Abramowitz and Stegun [1]. In particular, $K_{0}(z)$ has a logarithmic singularity at the origin,

$$
K_{0}(z) \sim-\ln z \quad \text { as } z \rightarrow 0,
$$

and is exponentially decaying for large $z$,

$$
K_{0}(z) \sim \sqrt{\frac{\pi}{2 z}} e^{-z} \text { as }|z| \rightarrow \infty .
$$

For $\lambda<0$, a particular solution of (2.18a) is, from [10],

$$
-\frac{1}{4} Y_{0}\left((-\lambda)^{1 / 2} r\right)
$$

This solution does not satisfy the radiation condition (2.18c) and must be augmented by the complementary solution

$$
\sum_{m=1}^{\infty} a_{m} J_{m}\left((-\lambda)^{1 / 2} r\right) \cos m \theta
$$

where $\theta=\tan ^{-1}(y-\eta / x-\xi)$. This is achieved by considering the asymptotic expressions of these Bessel functions for large $r$, and choosing constants $a_{m}$ so that the infinite sum cancels the $Y_{0}$ term on the upstream half plane $(x \rightarrow-\infty)$. We thus arrive at the solution for $\lambda<0$,

$$
\begin{aligned}
& G(x, y ; \xi, \eta) \\
& \quad-\frac{1}{4}\left[Y_{0}\left((-\lambda)^{1 / 2} r\right)+\frac{4}{\pi} \sum_{n=1}^{\infty} J_{2 n-1}\left((-\lambda)^{1 / 2} r\right) \cos [(2 n-1) \theta] /(2 n-1)\right] .
\end{aligned}
$$

The infinite sum in this equation may also be written [1]

$$
\sum_{n=1}^{\infty}()=-\frac{1}{2} \int_{\pi / 2}^{\theta} \sin \left(z \sin \theta^{\prime}\right) d \theta^{\prime},
$$

where $z=(-\lambda)^{1 / 2} r$, and which is evaluated numerically. Far upstream, $\theta \rightarrow \pi$, $z \rightarrow \infty$; this expression behaves like $(\pi / 2) Y_{0}(z)$, thus verifying the cancellation of upstream waves. Far downstream, $\theta \rightarrow 0, z \rightarrow \infty$, this expression behaves like $(\pi / 2) Y_{0}(z)$, and the Green's function in equation (2.21) becomes

$$
G(x, y ; \xi, \eta) \sim-\frac{1}{2} Y_{0}\left((-\lambda)^{1 / 2} r\right) \text { as } \theta \rightarrow 0 \text { and } r \rightarrow \infty .
$$

At the origin this solution has the same logarithmic singularity as equation (2.20), and the final solution in equation (2.19) must therefore be treated as an improper integral. This is a generalized solution for the two-layer flow over arbitrary topography, $h(x, y)$. In the solutions that follow, the infinite sum in 
(2.21) was evaluated by means of a Simpson rule estimation of the integral in (2.22), and the integral in (2.19) using a four-point numerical quadrature (Abramowitz and Stegun [1], page 892).

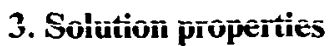

In each layer the flow is determined by a linear combination of the two fundamental modes (equations (2.16)), whose properties are dependent on the sign of $\lambda$. Thus the flow may consist of the sum of two evanescent modes (equation (2.20)), two wavelike modes (equation (2.21)) or a combination of an evanescent and a wave-like mode. Conditions on which of these combinations occurs are described by McCartney [8], and are largely dependent on the signs of $U_{1}$ and $U_{2}$. More general conditions are derived here.

Physical arguments suggest that if the flow in both layers is westward $\left(U_{1}<0\right.$, $U_{2}<0$ ), westward travelling Rossby waves which are generated will be swept downstream and the steady state will consist of an exponentially decaying disturbance which corresponds to the first case above. However, if eastward flow exists in at least one of the layers, westward propagating Rossby waves are retarded and the ultimate steady state will involve some stationary wave-like character.

In order to examine the individual modes more closely it is convenient to write equation (2.14) in terms of $\lambda$ (from equation 2.12). Thus:

$$
\lambda^{2}-\left(\gamma_{1}+\gamma_{2}\right) \lambda+\left(\gamma_{1} \gamma_{2}-F_{1} F_{2}\right)=0,
$$

with roots

$$
\lambda^{(1),(2)}=\left(\gamma_{1}+\gamma_{2}\right) / 2 \pm\left[\left(\gamma_{1}-\gamma_{2}\right)^{2} / 4+F_{1} F_{2}\right]^{1 / 2} .
$$

Since the term in the square bracket is positive definite, two real roots of equation (3.2) always exist. Graphs of

$$
G^{\prime}(\lambda)=\lambda^{2}-\left(\gamma_{1}+\gamma_{2}\right) \lambda+\left(\gamma_{1} \gamma_{2}-F_{1} F_{2}\right),
$$

for $\gamma_{1} \gamma_{2}-F_{1} F_{2}=-\beta\left(F_{1} U_{2}+F_{2} U_{1}-\beta\right) / U_{1} U_{2}<0$ are drawn in Figure 2. The two cases depicted correspond to $\gamma_{1}+\gamma_{2}>0$ and $\gamma_{1}+\gamma_{2}<0$, implying that $\lambda^{(1)}>0$ and $\lambda^{(2)}<0$. Disturbances in each layer therefore comprise a combination of an evanescent mode and a stationary wave-like mode. 


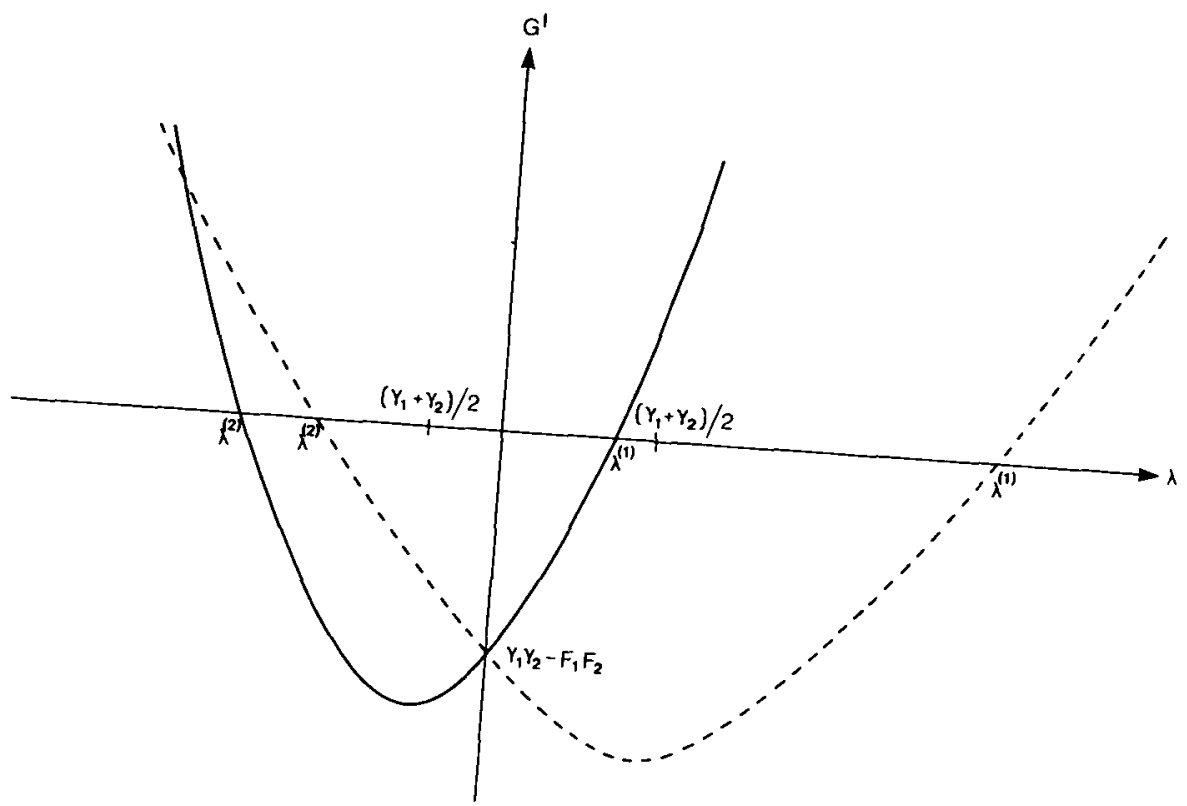

Figure 2. Graph of $G^{\prime}$ (equation (3.3)) with $\beta=2, F_{1}=0.52, F_{2}=1.22$ and $\gamma_{1}+\gamma_{2}<0$ (solid curve), $\gamma_{1}+\gamma_{2}>0$ (broken curve).

For fixed $\beta, F_{1}$ and $F_{2}$ the straight line

$$
S\left(U_{1}, U_{2}\right)=F_{1} U_{2}+F_{2} U_{1}-\beta=0
$$

divides the $\left(U_{1}, U_{2}\right)$ plane into physically distinct regimes.This is shown in Figure 3 for $\beta=2.9, F_{1}=0.52$ and $F_{2}=1.22$ which are typical oceanic and atmospheric values.

In region $\mathrm{A}$, where $\gamma_{1} \gamma_{2}-F_{1} F_{2}>0$, both $\lambda^{(1)}$ and $\lambda^{(2)}$ are negative and two wave-like modes exist. In region $B$, where $\gamma_{1} \gamma_{2}-F_{1} F_{2}<0$, at least one of the basic flows is eastward, and $\lambda^{(1)}>0, \lambda^{(2)}<0$ implying a combination of an evanescent and a wave-like mode. From equations (2.16) and (2.19) it follows that the contribution to the upper layer flow from each of these modes is the same and equal to $\alpha^{(1)} \alpha^{(2)} /\left(\alpha^{(1)}-\alpha^{(2)}\right)$, whereas in the lower layer the amplitude of the evanescent mode is $\alpha^{(1)} /\left(\alpha^{(1)}-\alpha^{(2)}\right)$ and that of the wave-like mode is $\alpha^{(2)} /\left(\alpha^{(1)}-\alpha^{(2)}\right)$. Thus the relative amplitudes of these modes in each layer over the bump is controlled by the values of $\alpha^{(1)}$ and $\alpha^{(2)}$ which are shown as functions of $U_{1}$ and $U_{2}$ in Figure 4.

In the counter-flow situation it can be seen, for example, if the lower layer flow is eastward and small $\left(U_{2} \ll \beta / F_{1}\right),\left|\alpha^{(2)}\right| \gg\left|\alpha^{(1)}\right|$ and the lower layer flow is dominated by the wave-like mode. On the other hand if the upper layer flow is eastward and small $\left(U_{1} \ll \beta / F_{2}\right),\left|\alpha^{(1)}\right| \gg\left|\alpha^{(2)}\right|$ and the lower layer flow is dominated by the evanescent mode. 


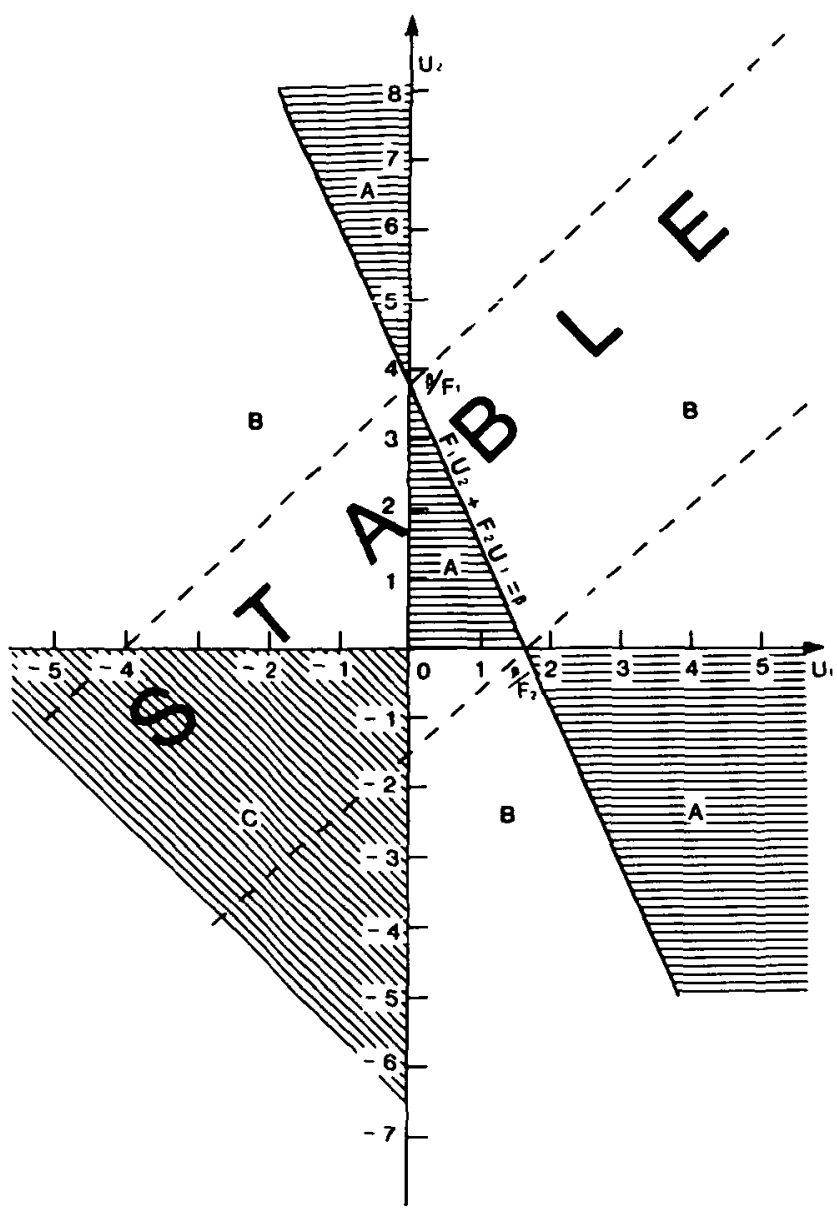

Figure 3. Solution regimes in the $\left(U_{1}, U_{2}\right)$ plane for parameter values given in Figure 2. Regions marked are A: two wave-like modes; B: one wave-like and one evanescent mode; C: two evanescent modes.

Far downstream of the topographic bump, where the evanescent mode has decayed, the flow in each layer is wave-like and the ratio of the upper layer disturbance to the lower layer disturbance is $-\alpha^{(1)}$. Thus areas of region $B$ where $\left|\alpha^{(1)}\right| \gg 1$ correspond to situations where the topographic influence far downstream of the bump is confined to the upper layer only.

In region $\mathrm{C}$, the flow in both layers is westward $\left(U_{1}<0, U_{2}<0\right)$ and both $\lambda^{(1)}$ and $\lambda^{(2)}$ are positive, which implies two evanescent modes verifying the previous physical argument. 

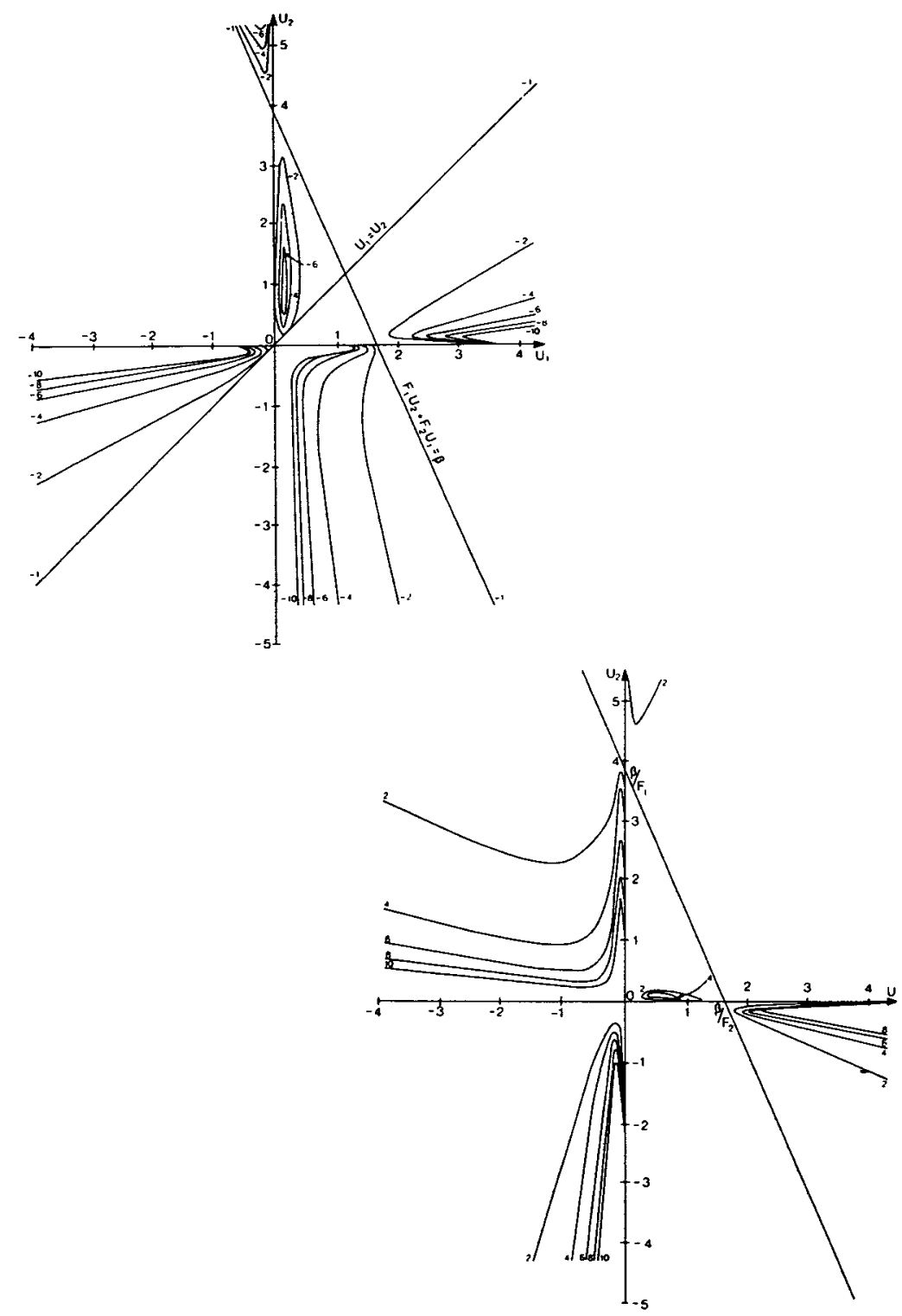

Figure 4. Contours of (a) $\alpha^{(1)}$ and (b) $\alpha^{(2)}$ in $\left(U_{1}, U_{2}\right)$ space for same parameter values given in previous figures.

\subsection{Equal upper and lower layer flows}

In the special case $U_{1}=U_{2}=U$, it is easily found from equations (2.12) and (2.15) that

$$
\alpha^{(1)}=-1, \quad \alpha^{(2)}=F_{1} / F_{2},
$$


and

$$
\lambda^{(1)}=F_{1}+F_{2}-\beta / U, \quad \lambda^{(2)}=-\beta / U .
$$

Equations (2.16) then reduce to

$$
\begin{gathered}
\phi_{1}=\left(\Phi^{(2)}+F_{1} / F_{2} \Phi^{(1)}\right) /\left(1+F_{1} / F_{2}\right), \\
\phi_{2}=\left(\Phi^{(2)}-\Phi^{(1)}\right) /\left(1+F_{1} / F_{2}\right),
\end{gathered}
$$

indicating that the disturbance fields in each layer are composed of a barotropic mode $\Phi^{(2)}$ of wavenumber $(-\beta / U)^{1 / 2}$, and a baroclinic mode $\Phi^{(1)}$, whose amplitude ratio between the upper and lower layer is $-F_{1} / F_{2}=-D_{2} / D_{1}$.

A barotropic westward flow, $U<0$, is responsible for two evanescent modes; the barotropic one decaying exponentially on a scale of $(-\beta / U)^{-1 / 2}$, and the baroclinic one decaying on a scale of $\left(F_{1}+F_{2}-\beta / U\right)^{1 / 2}$. For relatively large stratification, or small westward flow, $-\beta / U \gg F_{1}+F_{2}$, and both modes are reminiscent of inertial boundary layer flow in which strict geostrophy breaks down and significant motions occur in a region of width $(-\beta / U)^{-1 / 2}$ along the boundary.

In the case $U>0, \lambda^{(2)}$ is negative and $\lambda^{(1)}$ may be positive or negative. Thus the barotropic mode is always wave-like. The baroclinic mode however is only wave-like if $0<U<\beta\left(F_{1}+F_{2}\right)$. If $U>\beta\left(F_{1}+F_{2}\right)$ the baroclinic mode is evanescent, in which case the disturbance fields far downstream of the topography are dominated by the wave-like barotropic mode with the consequence that the upper and lower layer flows are nearly equal.

\subsection{Stability analysis}

A stability analysis of this two-layer system was first carried out by Phillips [13], and some of the results are used here to show that if the magnitude of the shear speed $U_{s}=U_{1}-U_{2}$ exceeds some critical value, there exists a range of wavelengths for which the disturbance modes are baroclinically unstable.

Phillips showed that for disturbances of total wavenumber $K$, stability depended on the sign of the term

$$
Q=\beta^{2}\left(F_{1}+F_{2}\right)^{2}+2 \beta U_{2} K^{4}\left(F_{1}-F_{2}\right)-K^{4} U_{s}^{2}\left(4 F_{1} F_{2}-K^{4}\right) .
$$

The system is stable if $Q \geqslant 0$ and unstable if $Q<0$.

The equation $Q=0$ is a quadratic equation for $K^{4}$ with roots

$$
K^{4}=\left(2 \beta U_{s}\left(F_{2}-F_{1}\right)+4 U_{s}^{2} F_{1} F_{2} \pm\left[16 U_{s}^{2} F_{1} F_{2}\left(U_{s} F_{1}+\beta\right)\left(U_{s} F_{2}-\beta\right)\right]^{1 / 2}\right) / 2 U_{s}^{2} .
$$

It follows that if the shear occupies the range

$$
-\beta / F_{1} \leqslant U_{s} \leqslant \beta / F_{2}
$$


no real roots exist, $Q$ is always positive, and the system is stable at all wavenumbers. The region in $\left(U_{1}, U_{2}\right)$ space in which $\left(U_{1}, U_{2}\right)$ satisfy condition $(3.9)$ is shown in Figure 3.

Outside this region two distinct roots exist, $Q$ changes sign, and the basic flow will be unstable to disturbances of sufficiently small wavenumber. Equations (3.8) and (3.9) give the wavenumbers at minimum and maximum critical shear to be

$$
\begin{array}{ll}
K_{\min }^{2}=\left[F_{1}\left(F_{1}+F_{2}\right)\right]^{1 / 2}, & U_{s}=-\beta / F_{1}, \\
K_{\max }^{2}=\left[F_{2}\left(F_{1}+F_{2}\right)\right]^{1 / 2}, & U_{2}=\beta / F_{2} .
\end{array}
$$

Thus when the shear exceeds the critical limits defined in (3.9), disturbance modes are stable at all wavenumbers exceeding the respective critical limits defined in equation (3.10). If, for example, $F_{1}=F_{2}=F$ then for shears $\left|U_{s}\right|>\beta / F$, disturbance modes of wavenumbers $K^{2}>\sqrt{2} F$ are all stable.

\section{Specific examples}

Large scale atmospheric motions are characterized by the following scales:

$$
\begin{gathered}
L=1.0 \times 10^{6} \mathrm{~m}, \quad D_{1}=7000 \mathrm{~m}, \quad D_{2}=3000 \mathrm{~m}, \quad U=10 \mathrm{~ms}^{-1}, \\
f_{0}=0.6 \times 10^{-4} \mathrm{~s}^{-1}, \quad \beta_{0}=2 \times 10^{-11} \mathrm{~m}^{-1} \mathrm{~s}^{-1}, \quad g^{\prime}=1 \mathrm{~ms}^{-2},
\end{gathered}
$$

for which the non-dimensional parameters are given by

$$
\varepsilon=0.16, \quad \beta=2.0, \quad F_{1}=0.52, \quad F_{2}=1.22 \text {. }
$$

Large scale oceanic motions are represented by considerably smaller values of $\varepsilon$, $F_{1}$ and $F_{2}$, but the general solutions presented in the previous section are still applicable.

In order to illustrate the characteristics of the flow field the simple topographic feature given by

$$
h(x, y)=5 e^{-8\left(x^{2}+y^{2}\right)}
$$

is chosen. Similar studies previously mentioned derived solutions which were restricted to such simple topographic forms. However, in order to study realistic atmospheric or oceanic situations, more complicated topography is required, and this is easily incorporated using the general solution given by equation (2.19).

\subsection{Westward flow in both layers, $U_{1}=-1, U_{2}=-1$}

This case has already been discussed in Section 3.1, where it was shown that the motion consisted of a sum of an evanescent barotropic mode and an evanescent baroclinic mode. 


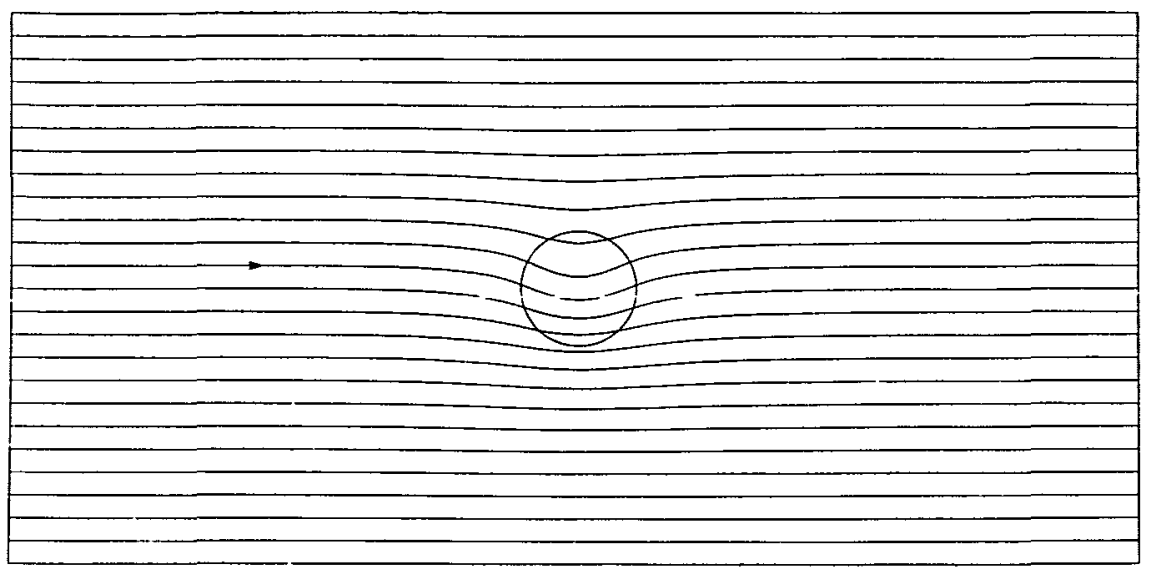

Figure 5. Lower layer streamlines for westward flow in both layers, $U_{1}=-1, U_{2}=-1$. Contour interval is 0.2 , and the nondimensional diameter of the circle is 1.0 .

The lower layer flow is shown in Figure 5. Topographic effects are confined to the immediate vicinity of the bump, with the flow deflecting equatorwards (due to the $\beta$-effect) as the bump is approached. In the upper layer, effects of stratification cause the flow due to the baroclinic mode to oppose that due to the barotropic mode with the result that very little topographic influence exists. In the limit $F_{1}, F_{2} \rightarrow 0, \lambda^{(1)}=\lambda^{(2)}=\beta$ and the barotropic and baroclinic modes are equal and opposite in the upper layer. In this case topographic effects are confined to the lower layer and the interface acts as a rigid lid.

\subsection{Eastward flow in both layers, $U_{1}=1, U_{2}=1$}

In this particular case, reference to Figure 3 shows that both fundamental modes are wavelike, with $\lambda^{(1)}=-0.26, \lambda^{(2)}=-2$ and $\alpha^{(1)}=-1, \alpha^{(2)}=0.43$. The non-dimensional wavelength of the baroclinic mode $2 \pi /\left(-\lambda^{(1)}\right)^{1 / 2}=12.3$, and that of the barotropic mode $2 \pi /\left(-\lambda^{(2)}\right)^{1 / 2}=4.4$. From equations (2.12) and (2.16), it may be seen that the upper layer consists of equal, but opposite, combinations of these two modes, while in the lower layer the amplitude of the baroclinic mode is more than double that of the barotropic mode. The result is that the lower layer downstream wake is of larger amplitude than that of the upper layer and of much larger wavelength. Furthermore, the flow in the lower layer is deflected equatorwards over the topography (Figure 6), reflecting the dominance of the $\beta$-effect in the potential vorticity balance. If larger eastward flows persisted such that $F_{1} U_{2}+F_{2} U_{1}>\beta$, the upward deflection of the interface would overcome the $\beta$-effect with the consequence that the lower layer flow would deflect polewards as the topography is approached. Of special interest are 
the disturbances which occur if $U_{1} \gg \beta / F_{2}$ and $U_{2} \ll \beta / F_{1}$ or if $U_{1} \ll \beta / F_{2}$ and $U_{2} \gg \beta / F_{1}$. In these situations $\left|\alpha^{(1)}\right| \gg\left|\alpha^{(2)}\right|$ and the flow over the bump is dominated by the evanescent mode, and topographic effects are confined to the lower layer. Evidence of stationary Rossby waves vanishes in the neighbourhood of the bump and the disturbance is similar to that which occurs when westward flow exists in both layers.

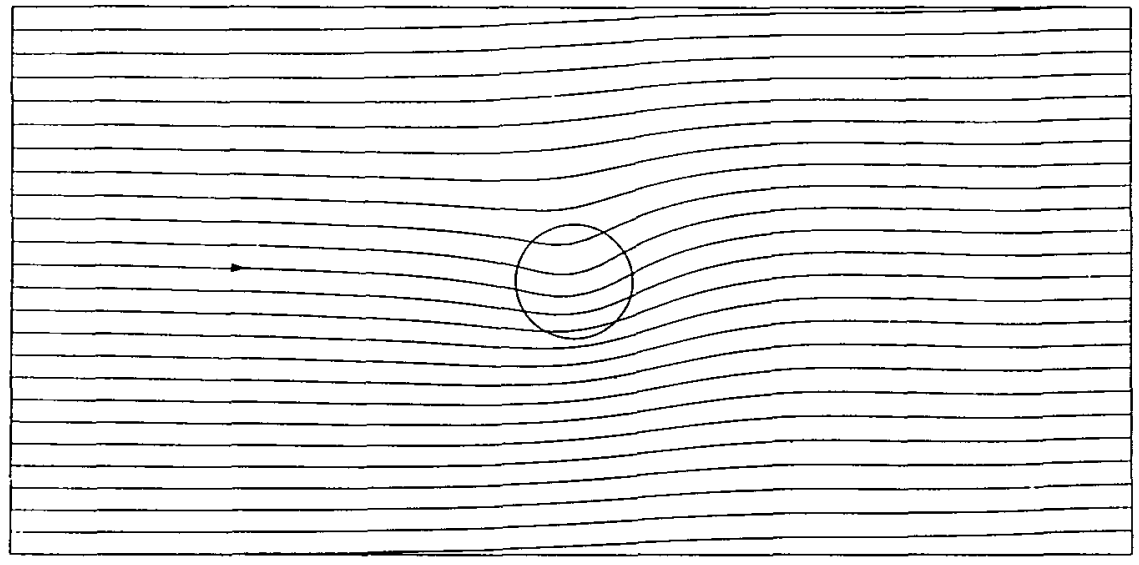

Figure 6. Lower layer streamlines for eastward flow in both layers, $U_{1}=1, U_{2}=1$.

Contour interval is 0.2 .

\subsection{Counter flow cases}

Most previous workers have neglected this situation as they have applied the theory to the ocean, where a total reversal in the direction of flow with height is rather rare. In the atmosphere, however, vertically sheared flows are common and the counter flow situation considered here has immediate application.

In the first example $U_{1}=-1$ and $U_{2}=1$, in which case $\lambda^{(1)}=1.6, \lambda^{(2)}=-3.3$ and $\alpha^{(1)}=-0.11, \alpha^{(2)}=4.01$. Thus the flow in both layers consists of an evanescent mode and a wave-like mode, and in the near downstream field the lower layer is dominated by the wave-like mode (Figure 7) with very little disturbance in the upper layer. In the general case of upper layer westward flow and lower layer eastward flow, reference to Figure 4 shows that in region $B$ the wave-like mode will always dominate the near downstream flow $\left(\left|\alpha^{(2)}\right|>\left|\alpha^{(1)}\right|\right)$ and the amplitude of disturbance will be greater in the lower layer. This case is further illustrated in Figure 8 which shows the lower layer flow when $U_{1}=-2, U_{2}=0.3$. The wave-like mode completely dominates the near downstream wake $\left(\left|\alpha^{(2)}\right| \gg\left|\alpha^{(1)}\right|\right)$ and the disturbance in the upper layer is negligibly small. Special features of the downstream flow in the lower layer are the strong south-eastward directed jet, with 
speed about $3 U_{1}$ along the north-eastern edge of the bump and a strong reversed flow to the south. The extensive meandering wake downstream of the bump is dynamically equivalent to the lee-wave field induced by an obstacle in a stratified fluid [10].

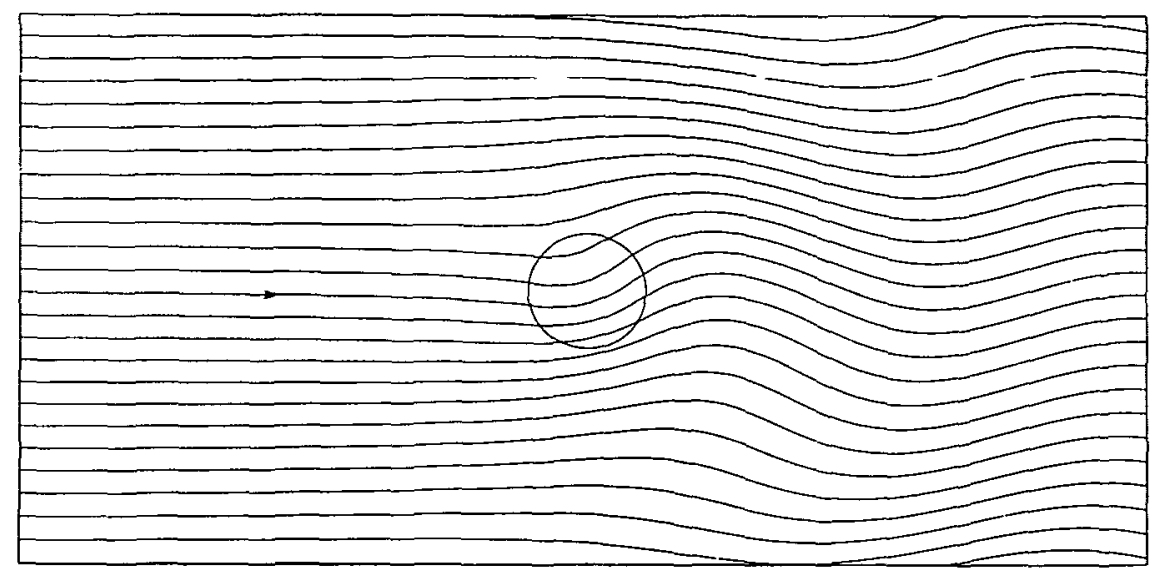

Figure 7. Lower layer streamlines for westward flow in the upper layer and eastward flow in the lower layer, $U_{1}=-1, U_{2}=1$. Contour interval is 0.2 .

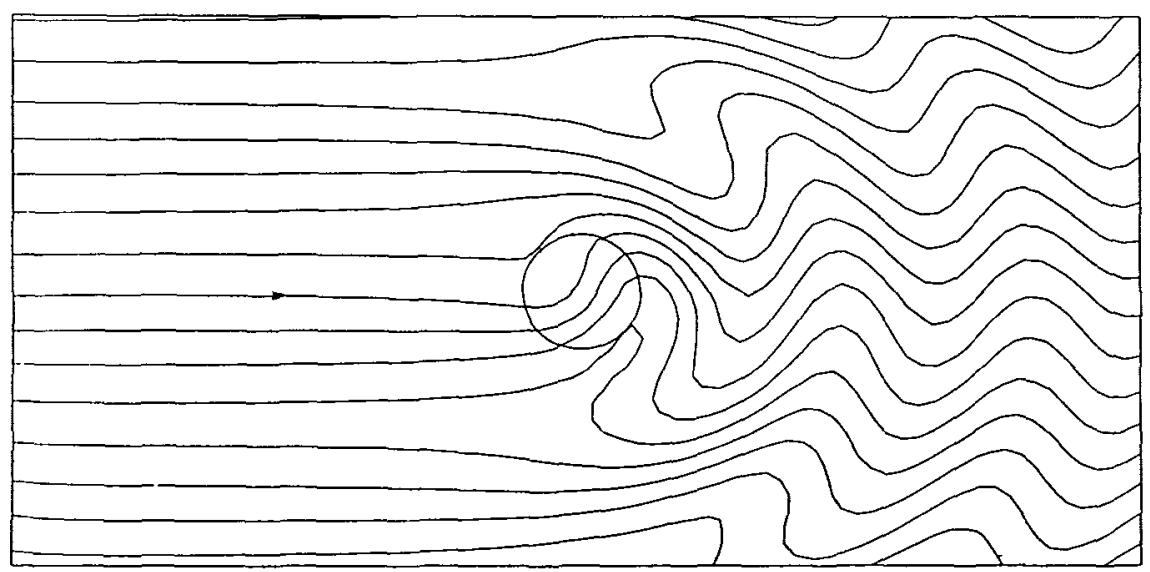

Figure 8. As in Figure 7 with $U_{1}=-2, U_{2}=0.3$. Contour interval is 0.1 .

If the lower layer eastward flow is large, $U_{2}>\beta / F_{1}$ and the upper layer westward flow small enough, $\left(-U_{1}\right)<\left(F_{1} U_{2}-\beta\right) / F_{2}$ disturbances in each layer comprise of two wave-like modes, but are dominated by the one of much greater wavelength.

In the third example we consider $U_{1}=1, U_{2}=-1$ in which case $\lambda^{(1)}=0.98$, $\lambda^{(2)}=-2.7$ and $\alpha^{(1)}=-2.9, \alpha^{(3)}=0.15$. Again the flow consists of an evanescent 
mode and a wave-like mode, with the near downstream disturbance much stronger in the lower layer. However, in this case the lower layer is dominated by the evanescent mode $\left(\left|\alpha^{(1)}\right|>\left|\alpha^{(2)}\right|\right)$ and the disturbance in this layer is nearly identical to that which occurs when the basic flow is westward in both layers (Figure 5). Thus, although a wave-like mode exists, its amplitude in the lower layer is negligible compared to that of the evanescent mode. This will always be the case provided the eastward flow in the upper layer is small enough, $U_{1}<\beta / F_{2}$ and the lower layer westward flow large enough $\left(-U_{2}\right)>\left(F_{2} U_{1}-\beta\right) / F_{1}$.

In each of these examples the upper layer disturbance in the vicinity of the bump is negligibly small, thus the absence of figures depicting the upper layer flow. Situations will occur, however, where the upper layer disturbance is of similar amplitude to the lower layer one, and these may be identified from Figure 4 when $\alpha^{(1)}$ and $\alpha^{(2)}$ are of similar orders of magnitude.

Although the case is of great interest, care is needed in the application of the counter-flow study to practical situations. For the baroclinic mode, the vertical wavelength of the standing waves is comparable with twice the total depth of the two layers. Thus in a practical situation where the interface between the two flows contains a critical layer (assumed viscous) and the interface thickness is comparable in height with the total depth of the flow, substantial dampening of the baroclinic mode may occur. For thinner interfaces such as that modelled here, tunnelling of wave energy occurs across the critical layer with little loss of energy as required by the present formulation.

\section{Conclusions}

A general solution for the vertically sheared, two-layer flow over an arbitrarily shaped bump on a $\beta$-plane has been found. Characteristics of the disturbances induced are determined principally by the configuration of the basic mean flows, the stratification parameters and the $\beta$-effect, and largely independent of the shape of the bump which only appears as a multiplying factor in the integral of equation (2.19). The amplitudes of the disturbances are directly proportional to the height of the obstacle for a given basic state.

Stationary waves forced by topography occur when there is eastward flow in at least one of the layers. In the case of eastward flow in both layers these are classical Rossby waves, and in the counter-flow situation are topographically modified Rossby waves. These waves occur on the downstream (eastward) side of the bump. A radiation condition prevents wave energy travelling from upstream. The wake associated with these waves extends many obstacle widths downstream and decays like $O\left(r^{-1 / 2}\right)$, where $r$ is the radial distance from the obstacle. 
When the basic flow in both layers is westward, disturbances are evanescent and confined to the neighbourhood of the obstacle. For obstacles which are symmetric, the disturbances are symmetric in the upstream-downstream direction, and the flow is deflected equatorwards.

The theoretical model presented has applications to the flow over topographic ridges in both the atmosphere and ocean. Special emphasis has been given to the counter-flow situation which has relevance to atmospheric flows in the sub-tropics.

\section{References}

[1] M. Abramowitz and I.A. Stegun (eds.), Handbook of mathemattcal functions (Dover, New York, 1970)

[2] D. L. Boyer, "Rotating flow over a step", J. Fluid Mech. 50 (1971), 675-687.

[3] D. L. Boyer, "Two-layer rotating steady viscous flow over long ridges", J. Fluid Mech. 111 (1981), 1-14.

[4] C. B. Fandry and L. M. Leslie, "A two-year quasi-geostrophic model of summer trough formation in the Australian sub-tropical easterlies" (submitted)

[5] N. G. Hogg, "On the stratified Taylor column", J. Fluid Mech. 58 (1973), 517-537.

[6] H. E. Huppert, "Some remarks on the initiation of inertial Taylor columns", J. Fluid Mech. 67 (1975), 397-412.

[7] M. S. McCartney, "Taylor columns and Rossby wakes generated by isolated topographic features on a $\beta$-plane," Notes on the 1972 Summer Study Program in Geophysical Fluid Dynamics at the Woods Hole Oceanographic Institution, WHOI Ref. 72-79 (1972), 60-81.

[8] M. S. McCartney, "Inertial Taylor columns on a $\beta$-plane", J. Fluid Mech. 68 (1975), 71-95.

[9] M. S. McCartney, "The interaction of zonal currents with topography with applications to the Southern Ocean", Deep-Sea Res. 23 (1976), 413-427.

[10] J. W. Miles and H. E. Huppert, "Lee waves in a stratified flow. Part 2, Semi-circular obstacle". J. Fluid Mech. 33 (1968), 803-814.

[11] J. Pedlosky, "The stability of currents in the atmosphere and the oceans. Part I", J. Atmos. Sci. 27 (1964), 201-219.

[12] J. Pedlosky, Geophysical flutd dynumics (Springer-Verlag, New York, 1979).

[13] N. A. Phillips, "Energy transformations and meridional circulations associated with simple baroclinic waves in a two-level, quasi-geostrophic model", Tellus 6 (1954), 273-286.

[14] G. H. Porter and M. Rattray, "The influence of variable depth on steady zonal barotropic flow", Deutsch. Hydrogr. Zeit. 17 (1964), 164-174.

[15] G I. Taylor, "Experiments on the motion of solid bodies in rotating fluids", Proc. Roy. Sci. London Ser. A 104 (1923), 213-218. 